\title{
Modelos de educación moral y víctimas
}

\author{
Moral education models and victims
}

Xabier Etxeberria

Universidad de Deusto

xetxemau@deusto.es

doi: http://dx.doi.org/10.18543/djhr.1759

Fecha de recepción: 24.01.2020

Fecha de aceptación: 08.04.2020

Cómo citar/Citation: Etxeberria, Xabier. 2020. «Modelos de educación moral y víctimas». Deusto Journal of Human Rights, n. 5: 41-68. doi: http://dx.doi.org/10.18543/djhr.1759

Sumario: Introducción. 1. Abrir la educación a las víctimas. 1.1. Las víctimas en las aulas. 1.2. El modo de presencia de las víctimas. 2. Modelos de educación moral. 3. El modelo de la alteridad y las víctimas. 3.1. El enfoque de la alteridad. 3.2. El enfoque levinasiano de alteridad y víctimas. 4. El modelo de la relación de cuidado y las víctimas. 4.1. El enfoque del cuidado. 4.2. Enfoque del cuidado y víctimas. 5. El modelo de educación del carácter moral y las víctimas. 5.1. El enfoque del carácter moral. 5.2. Enfoque del carácter moral y víctimas. 6 . El modelo del desarrollo del juicio moral y las víctimas. 6.1. El enfoque del juicio moral. 6.2. Enfoque del juicio moral y víctimas. Conclusión: el revulsivo transversal de las víctimas. Referencias bibliográficas.

Resumen: Ya desde la antigüedad griega se han formulado propuestas para la educación moral. Llama la atención que, a pesar de su pluralidad y del tiempo transcurrido, las víctimas no hayan tenido cabida significativa en ellas. Esto, además de alimentar una injusticia por implicar no reconocimiento de sus derechos, ha supuesto una merma grave en la calidad de dicha educación. En este trabajo se afronta tal carencia indagando cómo quedan transformados los modelos educativos hoy relevantes cuando se hace centralmente presentes a las víctimas en los diseños formativos de cada uno de ellos. Se tienen en consideración concretamente los enfoques clásicos de la formación del carácter y el desarrollo del juicio moral, y los más recientes de la relación de cuidado y la alteridad. Siempre con el horizonte de lograr una educación moral fundada y sólida que se sustenta en la recepción del impacto de las víctimas y acoge sus derechos.

Palabras clave: Educación moral, víctimas, receptividad, alteridad, relación, juicio moral, derechos humanos, virtudes. 
Abstract: Proposals for moral education have been formulated since as long back as in Greek Antiquity. It is remarkable that, despite its plurality and its long history, victims have rarely found a relevant space in that education. This fact does not only fuel an injustice, a non-recognition of their rights, but it also means a serious damage to the quality of such education. This paper seeks to fill in such a gap by looking into how contemporary educational models are improved when victims are considered in their respective formative designs. This transformation is dwelt upon here from the perspective of classical approaches such as the formation of character or the development of moral judgement, and also from more recent paradigms such as the care relation and alterity. Always keeping in mind the ultimate objective of achieving a wellfounded and sound moral education, this is pursued by never forgetting the impact on the victims and the protection of their rights.

Keywords: Moral education, victims, receptivity, alterity, relation, moral judgement, human rights, virtues. 


\section{Introducción}

Para guiar y alentar la educación moral, se han propuesto diversos modelos. Todos ellos tienen presupuestos específicos en torno a la concepción del ser humano, de la sociedad, de la moralidad. Ahora bien, tradicionalmente han tendido a compartir un enfoque central: eduquemos en la orientación a lo bueno aportando pautas y motivaciones que conduzcan a que se realice, en los educandos y en las sociedades; y a que, cuando esto no suceda, quede señalado el mal a evitar, para afrontarlo como proceda. En un enfoque como este las víctimas -las que sufren el mal-, si aparecen, lo hacen solo colateralmente.

Pues bien, estas líneas están motivadas por tres tesis, reactivas ante esta constatación. Primera: la difuminación de la presencia de las víctimas en la educación moral, por un lado, expresa por omisión una injusticia respecto a ellas como falta del reconocimiento en el sistema educativo al que tienen derecho (es decir, estamos ante una cuestión de derechos humanos) y, por otro, priva a los procesos de aprendizaje ético de un referente decisivo para lograr el objetivo que persiguen. Segunda: la presencia de las víctimas, que se impone por esas razones, solo es adecuada si es presencia de sujetos participantes en la dinámica educativa, no de objetos tenidos en cuenta, por respeto a ellas y como garantía de fecundidad. Tercera: esta presencia convulsiona positivamente los modelos de educación moral, purificándolos y reconfigurándolos, y forzándoles a interpelaciones y articulaciones fecundas entre ellos. Como se ve, no propongo un modelo alternativo a los tradicionales, que podría ser el de la alteridad (Ortega y Romero 2013). Invito más bien a que todos los modelos, los que aquí tendré presentes y otros que podrían considerarse, se dejen impactar receptivamente por iniciativas pedagógicas reales que impliquen esta presencia focal de las víctimas y acojan el reto de asumir las transformaciones que tal presencia les reclame.

Para dar razón de estas tesis no haré una exposición pegada a ellas, sino que seguiré un proceso argumental que espero las avale. Comenzaré presentando a las víctimas convocadas a entrar en las escuelas, así como los modos en los que es educativamente necesario que entren. Y pasaré luego a exponer los modelos de educación moral que deben ser tenidos en cuenta: ofreceré una síntesis de cada uno, organizada con el criterio de que facilite que se confronte con la presencia de las víctimas, y expondré luego esa confrontación, especificando, sin exhaustividad, las transformaciones que genera. 


\section{Abrir la educación a las víctimas}

\subsection{Las víctimas en las aulas}

Las tesis que acabo de formular no las propongo como mero fruto de una reflexión, aunque evidentemente esta deba darse. Son el resultado al que nos ha conducido el empeño —en el que he estado implicado con otras personas, entre las que las víctimas ocupan un lugar decisivo- por enfrentarnos desde el sistema educativo a la legitimación de la violencia terrorista ligada al País Vasco (decisivamente la de ETA, aunque no solo) y fomentar la correspondiente conciencia cívica. Tal empeño ha acabado mostrando con contundencia que la presencia en las aulas, en cuanto educadoras, de las víctimas de esa violencia de intencionalidad política, es clave, es insoslayable para el objetivo perseguido y para la justicia que se les debe, por más que en la sociedad vasca fuera percibida por muchos, incluyendo a educadores, como muy problemática, por no decir rechazable. ${ }^{1}$

Creo que fenómenos análogos están sucediendo en nuestro sistema educativo desde otros sectores de víctimas causadas por otras modalidades de violencia, sin que la reflexión sobre los modelos de educación moral haya sido suficientemente impactada por ello. Quiero referirme, de forma especial, al acoso escolar. ${ }^{2}$ Cuando en la escuela se ha sido consciente de él en toda su crudeza, algo se ha transformado. Porque, en general, si en la educación moral aparecían - poco - el victimario y la víctima, eran situados fuera del aula. Pero ahora, ambos están dentro. No podemos ya ignorar al primero, pero —algo decisivo - no podemos ignorarlo desde la reclamación, en general silenciosa a su pesar, de su víctima, porque "está ahí», ante todos, forzada a convivir en el espacio de su victimación, espacio que nos corresponde gestionar a los educadores. Por eso puede ser tan estimuladora no solo para alentar la acogida que merece -en la que, a la vez que le hacemos justicia, todos nos educamos moralmente, somos educados por ella-, sino, además, para que integremos también a otros sectores de víctimas.

Porque, desgraciadamente, las víctimas no se reducen a esos colectivos. Hay muchos más. En unos casos, a primera vista, se

1 Se presenta esta experiencia en Etxeberria (2014a), y con un enfoque testimonial en Arana y García (2012). La estructuración de los presupuestos que la inspiraron se encuentra en Bilbao y Etxeberria (2005).

2 También se están haciendo experiencias relevantes con víctimas de la violencia de género. 
mostrarán «externos» a los sujetos implicados directamente en la educación. En otros, estarán dentro. Pero incluso respecto a los que están fuera, podrán detectarse sus huellas en los que están dentro, en los estudiantes que son víctimas de los prejuicios discriminatorios - que pueden ser compartidos por compañeros suyos- e incluso de los comportamientos de violentadores externos. Piénsese para ejemplificar esto en tres sectores más de víctimas: las de la violencia de género, las de motivación racista, las que sufren la violencia estructural. ${ }^{3}$ Hay, respecto a todos ellos, una gran victimación externa que debe encontrar su lugar en la escuela, como lo tienen que encontrar los colectivos antes citados, comenzando por no ignorar sus ecos internos. Nos toparemos así, pues, con diversos sectores de víctimas: todas deben ser consideradas iguales en cuanto víctimas y recibir la correspondiente solidaridad; aunque, en su especificidad, aparecerán diferencias en sus modalidades de victimación (Bilbao, de la Cruz, Sasía 2010), aportándonos interpelaciones específicas que son relevantes para el aprendizaje educativo con el que enfrentarnos a ellas.

Los colectivos de víctimas mencionados hasta ahora lo son por una iniciativa de otros seres humanos que supone una injusticia. Pero la palabra "víctima» cubre un espectro más amplio, abarcando a toda persona que sufre. Se es también víctima, por ejemplo, de una inundación. ${ }^{4}$ A la educación moral se le impone a este respecto una distinción clave, para que no quede contaminada de inmoralidad, entre víctimas enmarcadas en el infortunio en sus relaciones con la naturaleza o con otras personas y víctimas causadas por la acción violenta intencional injusta, directa o estructural, de otros humanos. Es una distinción que, argumentalmente, se apoya en un criterio con presencia clave en el modelo de educación del juicio moral, pero que, existencialmente, se nos impone cuando contemplamos sin defensas prejuiciadas a las víctimas.

Comento un poco esto, anticipando parcialmente la conexión entre presencia de víctimas y modelos de educación moral. El criterio para hacer la distinción entre un tipo y otro de víctimas es el kantiano, afinado en lo que precise (Etxeberria 2014b), de la dignidad humana,

3 Como es evidente, por poner un caso cercano a la escuela, en el fortísimo impacto de la crisis económica en la salud psíquica y mental y en las capacidades cognitivas de los niños gravemente afectados por ella, muy numerosos.

4 Presupongo aquí que la victimación es solo consecuencia de la inundación (algo, reconozco, no frecuente), que, por tanto, no hay implicación de violencias estructurales que fuerzan a los pobres a vivir en condiciones mucho más expuestas al impacto destructor de la lluvia torrencial. 
que exige que nunca tratemos a ningún ser humano como puro medio, que respetemos sus derechos emanados de esa dignidad. La comprensión del criterio, su aplicación a situaciones de incertidumbre, está en principio arropada por los supuestos y procesos argumentales planteados en el modelo de educación del juicio moral. En este sentido, ese modelo ofrece un precioso aporte a todos los demás, que, a su modo, tendrán que integrarlo para quedar validados. Según él, se impone la distinción señalada entre víctimas del infortunio y víctimas de la causalidad intencional injusta humana. Por supuesto, para acogerlas educativamente a todas. Pero, comento por mi parte, planteando diferencias clave en esa acogida: las primeras víctimas nos convocan a la responsabilidad por lo que hay que hacer para superar en lo posible su sufrimiento; las segundas, además de convocar de ese modo a todos los no causantes de su dolor, convocan a una responsabilidad especial a los que se lo causaron o lo aprobaron, no meramente ante lo que hay que hacer, sino por lo que hicieron o consintieron. Es una responsabilidad que mira al pasado de su forzamiento de la dignidad del otro, reclamándoles, para empezar, que lo reconozcan, más allá de las excusas justificatorias que se hubieran formulado, como ha sido el caso del terrorismo; que lo reconozcan ante sus víctimas, expresando su dolor por ello, su deseo de que no hubiera pasado, su esfuerzo por reparar lo reparable.

Fijémonos ya por dónde quiero avanzar. Por un lado, trabajar argumentalmente el correcto juicio moral en relación con la justicia, según propone uno de los modelos de educación moral, se muestra necesario para que las víctimas sean adecuadamente situadas. Por otro, ese modelo, resituado desde las víctimas, queda de algún modo desbordado y transformado. Tras empujar a la correspondiente distinción de responsabilidades ante unas víctimas y otras, el modelo, especialmente ante las víctimas con victimadores, se ve convocado a un doble reto: a hacerse cargo del pasado de la memoria y no solo del presente de la iniciativa o el futuro de la promesa; y a incluir a la víctima no solo como referencia objetiva para el juicio de los educandos sino como copartícipe de él. Una breve observación sobre esto último, confirmada por la iniciativa que hemos hecho con las víctimas del terrorismo: cuando el alumnado está mínimamente desbloqueado de prejuicios que le impiden verlas como tales, el mero hecho de tenerlas delante y de escuchar el testimonio moral de su victimación le remite intuitivamente al principio de que nuestra dignidad es inviolable, pero a un principio que se muestra encarnado, con una intensidad afectiva que no solo no lo nubla sino que lo orienta hacia la convicción personal. Con lo que las suspicacias 
«sistemáticas» a la presencia de lo afectivo, propias de este modelo, quedan cuestionadas.

Dejo, de todos modos, para más adelante, el avanzar por este terreno del impacto de la víctima en los planteamientos de los modelos. Ahora, en esta llamada inicial a que estén presentes en los procesos de educación moral, lo que he querido subrayar con la distinción precedente es que hay que asegurarla, a fin de que esa educación no se contamine. Porque suele haber una fuerte tendencia, por parte de quienes victiman o se identifican con ellos, a fomentar dos estrategias que diluyen sus responsabilidades específicas: la de «naturalizar» su violencia, considerándola fruto de causalidades naturales ajenas a la intencionalidad humana (es lo habitual en la violencia estructural: parecería que mandan «los mercados» como manda la ley de la gravedad); y la de suprimir la distinción englobando a todas las víctimas en el concepto de sufrientes (con lo que el terrorista que es víctima de que le estalle la bomba que iba a colocar a una persona es sumado indistintamente con la víctima de otro terrorista que sufrió el impacto de la bomba que este le colocó; suma que al final diluye a las víctimas; y que pone en sordina inmoral el hecho de que el primero es víctima de sí mismo, no de una injusticia). Es cierto que hay a veces zonas oscuras para distinguir entre estos tipos de víctimas (¿hubo causalidad humana por negligencia de los responsables políticos en que una lluvia torrencial derivara en inundación destructora?), pero esto no pide suprimir la distinción sino aplicarla con discernimiento. Pues bien, quien pretenda fomentar la educación moral en los centros educativos implicando focalmente a las víctimas tiene que estar atento a no dejarse embaucar por ninguna de estas estrategias.

\subsection{El modo de presencia de las víctimas}

Las víctimas, pues, están convocadas a las aulas, pero: en su condición de víctimas según los criterios de los derechos humanos; y para ejercer una función educativa a través del testimonio moral de la victimación sufrida y de su modo de afrontarla.

Esto supone, para empezar: que las posibles presencias implícitas tienen que hacerse explícitas y centrales; que las focalizaciones que puedan haberse dado, al tratar el mal, en los victimarios, como ha sido común en la educación para la paz (Etxeberria 2013), tienen que transformarse en focalizaciones en las víctimas, desde las cuales hay que acercarse a los violentos y la maldad; y que la posible presencia pasiva de las víctimas, como «objeto» no puramente instrumental pero 
sí como aquello que se atiende, tiene que transformarse en presencia activa de sujeto.

Esta presencia puede ser física o virtual. En el primer caso, cuando la víctima está ante nosotros, es impensable que no sea activa, creativa. Su interpelación es en principio mucho más impactante, y también más delicada. Lo primero que debe garantizarse es que su intervención no solo no la revictimice sino que contribuya a su consistencia personal, a su sanación. Para lo cual, el centro escolar como tal y los profesores, tras asegurarse de que la víctima ha realizado un suficiente proceso de duelo como para afrontar el reto de su testimonio, deben ofrecerle condiciones dignas y empáticas de acogida. Todo esto se plantea de modo diferente en función de la persona, pero también en función de la victimación sufrida: es muy difícilmente pensable pedir a un niño o adolescente que testimonie el acoso que está sufriendo, pero cabe pedírselo al joven que ya lo ha encajado suficientemente; una víctima del terrorismo puede estar en disposición personal de intervención educativa, pero en el centro puede haber empatías con los victimarios que reclaman que se programe cuidadosamente su intervención. Considérese algo similar, pero acomodado a su especificidad, para las víctimas de la violencia de género, o racista, o estructural, etc.

La víctima, a su vez, se compromete a una intervención educadora, lo que supone que va a dar cuenta moral de lo que le ha pasado y de su reacción ante lo que le ha pasado. Esto excluye testimonios partidistas de su victimación y propuestas reactivas ante los violentos no acordes con los derechos humanos, por ejemplo, con petición de pena de muerte. La partidarización, el ligar el testimonio personal con la crítica de determinadas opciones democráticas, puede darse en víctimas de motivación política: es algo legítimo, pero su lugar no está en la escuela sino en el debate público. ${ }^{5}$ Todo esto pide convocar previamente a las víctimas predispuestas para estas intervenciones, dialogar con ellas sobre estas cuestiones, etc. El que estos procesos los vivan dentro de un grupo les ayuda enormemente. Mi experiencia personal es que las víctimas así convocadas a las aulas se expresan con una gran finura moral que integra ajustadamente la indignación al juzgar lo sucedido y que se abre a experiencias de afrontamiento

5 En la experiencia con víctimas del terrorismo esta ha sido una cuestión muy importante. Se ha afrontado desbordando sus supuestos mínimos. Dado que una víctima de ETA y una del GAL, desde el punto de vista moral, son ambas iguales, se ha producido hermanamiento entre ellas, capacidad de una para representar a la otra en las aulas. Lo que ha tenido una gran repercusión educativa. 
de la violencia enormemente ricas... y educativamente impactantes en quienes les escuchan.

Junto a la presencia física de las víctimas hay que estar abiertos a presencias virtuales, a través de la grabación de sus testimonios en soportes técnicos visuales, sonoros o escritos. Abren a posibilidades importantes: la generalización educativa de su presencia, la posibilidad de trabajos pedagógicos variados, etc. Pero se corre el riesgo de que al mostrarse en "objetos», ellas también acaben objetivadas de una cierta forma, sean «objeto de estudio» que, de algún modo, es domesticado por el profesor. Hay que trabajar todo lo posible para que la confección de esas presencias virtuales y la práctica educativa con ellas sean de tal calidad que la interpelación subjetiva de la víctima se mantenga viva. En estas presencias virtuales pueden incluirse relatos que son formalmente de ficción, pero que narran las vivencias de las víctimas de tal modo que muestran la verdad de lo que pasa en las violencias a las que remiten. Lo que nunca deberá hacerse es apelar a las riquezas de las presencias virtuales para frenar las presencias físicas.

Proyectar la presencia de las víctimas en la educación, en especial si es presencia directa, supone toda una preparación específica por parte de los responsables de los centros y de los profesores: para garantizarles una buena acogida y acompañamiento, para ayudarles en los previos que precisen conocer antes de su intervención, para situar esta intervención en un proceso pedagógico amplio, que queda todo él impregnado de esos momentos centrales de presencia, y que es diseñado y coordinado por los profesores en diálogo con las víctimas. No se podrá hacer participar a todos los sectores de víctimas, pero sí se podrá transmitir el mensaje de que, en lo más básico, las víctimas presentes han representado a las ausentes. Cuando la presencia es virtual toda la planificación queda por supuesto en manos del profesorado. ${ }^{6}$

\section{Modelos de educación moral}

En un texto ya lejano, Puig Rovira (1995) distinguía la vigencia de cuatro modelos de educación moral —para hacer luego su propio planteamiento: 1) como socialización; 2) como clarificación de valores; 3) como desarrollo del juicio moral; 4) como formación de hábitos virtuosos. ¿Son todos válidos? ¿Deben contemplarse otros?

6 Puede verse un diseño pedagógico para esta modalidad virtual de presencia, por lo que se refiere a las víctimas del terrorismo, en Harillo, Prieto y Ugarte (2006). 
Desde la perspectiva asumida en estas líneas, proyectar el foco de la presencia de las víctimas en ellos se convierte en criterio decisivo de discernimiento sobre su legitimidad de arranque: el modelo que, por lo que es en sí, bloquee la presencia de las víctimas por incubar la posibilidad de hacerlas irreconocibles, deja de ser admisible. Es lo que ocurre con los dos primeros, al menos si son concebidos como los presenta Puig Rovira, que quedan así totalmente convulsionados por el horizonte de presencia de las víctimas. En efecto, si el modelo de socialización presupone como tal educar para la adaptación acrítica de la conducta de las personas a los valores y las normas dominantes de la sociedad a la que se pertenece, en él se halla implicada la posibilidad de aceptar como legítima la victimación que tales normas pueden contener, por ejemplo, en forma de discriminación por razón de género.

Algo parecido puede decirse del modelo de clarificación de valores, en el que se trata de lograr que el educando sea lúcido respecto a los valores que posee, a fin de que ajuste coherentemente a ellos su conducta y, de ese modo, avance armonizadamente hacia su vida lograda. Aquí hay reconocimiento de autonomía, aunque en su versión más individualista y no con el rigor ético que le exige Kant (1973). El problema de la propuesta está no solo en que parecen ignorarse los arraigos culturales y sociales, ante los que no hay que tener actitudes sumisas como en el modelo anterior pero que se impone reconocer, sino en que —es lo que importa destacar aquíen ese cierre relativista del individuo en sus propios valores vuelve a ser posible asumir expresiones de ellos que justifiquen la victimación, por ejemplo, la que se da a través de estructuras económicas que se ven positivas porque se rigen, incluso en lo relativo a bienes que cubren las necesidades básicas, por una pura competitividad vista como valor.

Los otros dos modelos de educación moral, iluminados con el foco de las víctimas, ofrecen a la vez riquezas y puntos débiles, como trataré de mostrar cuando los aborde, pero no implican bloqueos previos como los precedentes. Tienen, por eso, una legitimación de partida, que en modo alguno debe inhibir la necesaria crítica interna. ¿Hay que postular más modelos que quepa considerar abiertos a la presencia interpeladora de las víctimas? En las últimas décadas han ido surgiendo algunos que, aunque no tan extendidos, son ética y pedagógicamente sugerentes y cubren este criterio. Me refiero concretamente al modelo de alteridad, o educación moral centrada en el otro, y al focalizado en la relación de cuidado. En lo que sigue voy a tener en consideración estos cuatro modelos, para confrontarlos con el reto de la presencia activa y central en ellos de las víctimas. 


\section{El modelo de la alteridad y las víctimas}

\subsection{El enfoque de la alteridad}

A primera vista, el modelo de educación moral que parece mostrarse más adecuado para incluir centralmente a las víctimas es el de la alteridad. Hasta podría presuponérsele inspirado por ellas. Hay aún pocos estudiosos que lo proponen expresamente como alternativa a los otros. ${ }^{7}$ Por mi parte, voy a presentarlo remitiéndome directamente a su inspirador principal, Levinas.

Según este autor, el dinamismo básico de las relaciones humanas debe situarse en la imbricación entre la exposición del otro - puro rostro o manifestación indefensa - ante el uno mismo, y la del uno mismo — pura pasividad — ante el otro.

— «El rostro está expuesto, amenazado, como incitándonos a un acto de violencia. Al mismo tiempo, el rostro es lo que nos prohíbe matar» (Levinas 1991b, 81). El otro es aquel cuyo sentido consiste en decirme, a la manera de una «extraña autoridad desarmada», que no ejerza violencia. "Hay, en la aparición del rostro, un mandamiento, como si un amo me hablase. Sin embargo, al mismo tiempo, el rostro del otro está desprotegido; es el pobre por el que yo puedo todo y a quien todo debo» (Levinas 1991b, 83).

- A su vez, el uno mismo está convocado a la pasividad desnuda radical, para que le impacte con toda fuerza ese rostro: «El uno [el yo, despojado de la priorización de su intencionalidad y autonomía que le constituyen en sujeto en sentido denso] se expone al otro como una piel se expone a aquello que la hiere, como una mejilla ofrecida a quien la abofetea» (Levinas 1987, 102). Se muestra como vulnerabilidad ante ese otro.

- Es en el despertar así de este "uno» en donde se constituye no la ética como sistema, sino «la intriga de la ética», plasmada por esa imbricada exposición mutua pero asimétrica, en la que el otro «interviene como traumatismo: esa es su manera peculiar» (Levinas 1994, 173).

7 Entre nosotros, Ortega (2004), Ortega y Mínguez (2005), Mínguez (2010), Ortega y Romero (2013), Ortega (2016), Mínguez, Romero y Pedreño (2016). También hay enfoque de alteridad en Bárcena (2003), y puede conexionarse con este modelo el texto de Santos (2012) inspirado en Benjamin y Adorno. 
En esta «intriga», al uno le es asignada la responsabilidad por el otro. Una responsabilidad que en su primer movimiento fundante es radical, pasiva: la que se expresa como disposición primaria e incondicional de obedecer el mandato del otro, "autoridad infinita», antes de que se formule. "La sujeción precede, en esta proximidad del rostro, a la decisión razonada de asumir la orden que lleva» (Levinas 1991c, 190). Pasividad de la responsabilidad que, aclara Levinas, "está más acá de la alternativa pasividad-actividad» comunes (Levinas 1987, 168), porque es en realidad la interrupción del egoísmo.

Una responsabilidad así planteada se nos muestra infinita: "soy responsable de aquel mismo que me ordena» (Levinas 1991a, 102), independientemente de la reacción que tenga hacia mí. Responsable hasta de su responsabilidad, como si su suerte estuviera en mis manos. Y ello configura mi identidad: «el psiquismo del alma es el otro en mí» (Levinas 1987, 126), mi ser es ser-para-el-otro. La unicidad del yo, en efecto, radica en el hecho de que nadie puede responder en mi lugar. Lo que implica una heteronomía radical, pero en la que el otro no me domina, sino que me «despierta» a la libertad para responder.

No cabe más limitación de esa responsabilidad que la exigida por la relación que debo establecer también con «los otros como el otro», que se me revelan en el otro y piden justicia. Esta aparece así con la entrada de esos otros - «el Tercero»- e introduce, en la directa relación asimétrica del cara a cara, las relaciones de simetría de los otros como el otro, entre los que yo, como otro, importa. La desmesura de la responsabilidad hacia el otro está llamada de este modo a conjugarse con la mesura de la relación de igualdad y reciprocidad entre los terceros, pero permaneciendo siempre la fuente de inspiración-llamada, pues el Tercero hacia el que se dirige la Justicia «me mira con los ojos del Otro». Es, pues, el olvido de sí el que mueve la justicia, no la coordinación de egoísmos.

Proyectemos esta propuesta a la educación moral, advirtiendo que Levinas no se deja aplicar fácilmente, pues pretende ir al corazón de la experiencia ética, al sentido de esta. Forzándole, por eso, algo ${ }^{8}$, el

8 Galanti (2013), comparando la propuesta de alteridad en Levinas (en especial la de 1987) y Ricoeur (1990), destaca muy bien cómo en el enfoque del primero hay un desequilibrio radical entre el otro y el sí mismo a favor del otro, dándose un encuentro como traumatismo ético —el impacto del otro en el sí mismo, en el que este percibe la llamada ética a la responsabilidad ilimitada-, en el cual la pasividad de este es total, impidiéndole que pueda vivenciar que la afección es recíproca. Todo esto hace muy difícil que quepa hablar de «relación intersubjetiva». En Levinas esta ética intersubjetiva, en forma de justicia, tiene su lugar propio con la aparición del «Tercero», de los otros como el otro. 
enfoque educativo de alteridad podría formularse así: 1) el punto de partida debe ser situado en la incentivación de la exposición asimétrica del otro ante «el yo» tal como ha sido descrita; en ella el que educa propiamente es el otro, mientras que el yo es el educado; 2 ) es educado en la medida en que se hace efectiva en él una pasividad-receptividad originaria ante ese otro; 3 ) en la que se sitúa su responsabilidad primaria, en la que deberán fundarse los otros movimientos responsabilizantes ligados a las deliberaciones que se sienta llamado a hacer; 4) su ser auténtico, que es su ser moral, se configura así como ser-para-elotro, del que responde, redefiniéndose el sentido de su heteronomía/ autonomía; 5) está convocado a abrirse a quienes desbordan su relación básica con el otro - los otros como el otro- para que se haga justicia con ellos, pero desde la expansión de la responsabilidad que surge de las exposiciones asimétricas. La relación con el otro se muestra así como una enseñanza, afirma el propio Levinas, añadiendo ajustadamente: pero no como mayéutica, porque lo decisivo no es lo que sale de uno mismo sino lo que entra a partir del otro. Los afectos están muy presentes, pero en la forma de ser afectados por el otro.

Una propuesta como esta se encarna más precisamente como educación moral si se concretan los sujetos implicados en ella: el otro, el sí mismo, el tercero. Ortega (2004) sitúa al otro -la referencia clave - en el educando como tal, siendo el educador ese "yo» que tiene que hacerse cargo de él. La educación es concebida como un acontecimiento ético, motivado por la aparición del otro que irrumpe sin previo aviso y al que no podemos dejar de mirar y responder.

«Desde la pedagogía de la alteridad, el proceso se inicia con la mutua aceptación y reconocimiento de maestro y alumno, en la voluntad de responder del otro por parte del profesor, en la acogida gratuita y desinteresada que presta al alumno de modo que este perciba que es alguien para el profesor y es reconocido en su singularidad personal. [...] En la pedagogía de la alteridad la acogida del otro significa sentirse reconocido, valorado, aceptado y querido por lo que uno es y en todo lo que es. Significa confianza, acompañamiento, guía y dirección, pero también aceptar ser enseñado por "el otro" (educando) que irrumpe en nuestra vida (educador)» (Ortega 2004, 11-12).

En esta aplicación de Levinas hecha por Ortega a la educación, apoyada en textos centrales del filósofo, junto a una interpelación educativa muy sugerente, detecto una ambigüedad: en sí se sitúa, evidentemente, en parámetros de alteridad levinasiana, pero en ocasiones la percibo orientada al modelo del cuidado, como en parte se desprende 
de la cita precedente. ¿Será porque para dinamizar decisivamente la alteridad se precisa un impacto contundente en el profesor, por ejemplo, el que se vea sorprendido por el descubrimiento de que un alumno suyo, sin que él se entere, ha sufrido un duro acoso escolar durante años? ¿Será que este es un modelo al que le es consustancial la presencia del otro víctima impactando tanto a profesores como a alumnos?

\subsection{Enfoque levinasiano de alteridad y víctimas}

Este enfoque, en efecto, parece aplicarse muy bien cuando hace su irrupción en el aula, invitado o no, externo o no, de modo físico o virtual, rompiendo la cotidianidad, alguien del que es manifiesta e impactante su condición de víctima: el otro expuesto de verdad ante profesores y alumnos que se exponen a su vez ante él. La dinámica indicada, profundamente educativa, empieza entonces por la fuerza de ese impacto. Podría concluirse, a partir de aquí, que el modelo solo vale para abordar las situaciones de victimación, pero deberá postularse más bien que lo aprendido en el afrontamiento de ellas debe "contagiar» todo acercamiento a lo moral, que vale en este sentido para todas las situaciones.

De todos modos, las experiencias impactantes reales con víctimas en las aulas, en especial con su presencia física, empujan a hacer matizaciones al acercamiento levinasiano, al menos visto como guía para la acción educativa. Paso a señalarlas.

En el otro levinasiano hay ciertas ambigüedades. A veces es presentado como el desamparado, el pobre, el violentado — la víctima-; otras como «el primero que llega». Y este primero que llega puede ser -lo reconoce el propio autor - el que me persigue injustamente; el rostro que percibo entonces es el del violentador. «En cierta medida también soy responsable de él», añade (Levinas 1991c, 123-124). Pues bien, las víctimas realmente presentes en las aulas, que «chocaron» a su pesar con sus otros victimadores, nos exigen que pautas educativas radicalmente diferentes marquen estos dos modos de otredad, diferenciados por la inocencia frente a la culpabilidad. Nos lo dicen incluso aquellas que está dispuestas a abrirse a la acogida de victimarios que se arrepientan. ${ }^{9}$

9 En este sentido, cuando conexionamos alteridad con compasión —como en Ortega (2016) o en Mínguez, Romero y Pedreña (2016)—, no tenemos que ignorar que la alteridad levinasiana, sobre todo la presente en De otro modo que ser (originalmente de 1974) en donde ya no se enfatiza a la «viuda y el huérfano» como prototipo del otro según se hacía en Totalidad e Infinito (obra de 1961), puede incluir al "otro perseguidor», lo que la complejiza y la distancia de su identificación con la compasión. 
En segundo lugar, la relación asimétrica que nos propone Levinas entre el otro y el uno carga muy densamente la asimetría, pero hace muy tenue la relación: el yo es convocado a su responsabilidad hacia el otro más allá de la reacción de este: "la recíproca es asunto suyo» (Levinas 1991b, 92). Las víctimas presentes en las aulas muestran palmariamente que la asimetría debe mantenerse, que el primer y decisivo momento para quien las escucha es el de la receptividad desprejuiciada. Pero muestran igualmente que es fundamental que aboque a un diálogo que crea relación real -como de hecho así sucede-, que para nada diluye la fuerza de la interpelación porque surge de ella, no frente a ella.

Por último, como señala Ricoeur (1990, 387-393), la hiperbolización levinasiana de la responsabilidad hacia el otro y de la definición del sí mismo a partir de ella, diluye las posibilidades del ajustado acercamiento al sí mismo definido por su anhelo de vida realizada unido a su capacidad de entablar relaciones recíprocas, simétricas cuando sea el caso. Las víctimas en las aulas no presuponen esta dilución, únicamente nos convocan a que la realización de sí como formando parte de la tarea moral, encuentre en la interpelación que emiten modos de afinarla... para plenificarla.

\section{El modelo de la relación de cuidado y las víctimas}

\subsection{El enfoque del cuidado}

Se fundamenta en la ética del cuidado que autoras como Gilligan, inspiradas en la historia de las mujeres adecuadamente discernida ante las condiciones de dominación sufridas, han elaborado. Y hay ya propuestas educativas consistentes como la de Noddings (2009) que asumo aquí como referencia. Se confronta sobre todo con la educación del carácter, a la que percibe cercana pero a la vez distinta. Considero, por cierto, que bastantes de las críticas que hace a esta provienen de una comprensión parcial y a veces incorrecta de ella, pero aquí me centraré en lo propositivo.

Los supuestos básicos del enfoque del cuidado pueden sintetizarse así: 1) para que el mundo mejore se precisan mejores personas, no mejores principios, por lo que el fin de la educación debe ser lo primero, en lo que debe enmarcarse lo segundo; 2) para lograrlo, no hay que focalizarse prioritariamente en la bondad del agente — virtuoso-, sino en las relaciones pertinentes entre agentes; 3) la modalidad relacional decisiva es la del cuidado — ser cuidados y cuidar - y el fomento de las 
virtudes debe darse en este marco; 4) también hay que focalizarse en la promoción de condiciones objetivas que, dentro y fuera de los centros educativos, favorecen la vida moral relacional; 5) estos objetivos implican una importante responsabilidad respecto al desarrollo moral de las personas con las que nos relacionamos que, con todo, no suple la responsabilidad de ellas.

En cuanto a las relaciones de cuidado: 1) aunque se distingue ser receptor de ser promotor del cuidado, ambos aportan y contribuyen a la relación; 2) la centralidad de las relaciones nos sitúa en nuestra condición primaria de interdependencia; 3) hay que priorizar el encuentro intersubjetivo sobre la identificación con la comunidad a la manera como se hace en el modelo de las virtudes, que tiene su lado oscuro del que hay que prevenirse, de forma que el cuidado surja de la necesidad del otro; 4) debe distinguirse cuidado natural, que emerge de la inclinación, de cuidado ético, al que recurrimos, reforzando el sentimiento, cuando nos resistimos al primero: «el estado preferido es el cuidado natural; el cuidado ético se invoca para restaurar el cuidado natural» (Noddings 2009, 41). En este sentido, la propuesta de relación de cuidado es una forma de naturalismo pragmático, que sitúa la fuente de la vida moral en la interacción humana real sustentada en la experiencia de ser cuidados; 5) la prolongación del cuidado hacia los lejanos - abrirse a la justicia - hay que alentarla a través de la expansión de la preocupación por los cercanos.

Al proyectar todo esto en la relación educativa vista como relación de cuidado (entre profesores y alumnos y entre alumnos), hay que tener presentes pautas como estas: 1) como destacó Hume, la motivación para el bien se encuentra en los afectos, no en las razones; 2) relevancia de las narraciones que ayuden a los educandos a entender lo que sucede en la vida real, incentivando con ellas la virtud de la compasión; 3) importancia del diálogo propio de la fenomenología del cuidado, el que, combatiendo la indiferencia ante el otro, se centra no en el debate en torno a temas sino en la atención a la subjetividad de los participantes; 4) ante problemas y fallos del educando, debe insistirse en alentarlo viendo lo mejor de él, en vez de condenar y castigar; 5) funcionará el ser modelos de conducta para los alumnos, pero sin que deba repararse en general en ello.

El horizonte de fondo de todo esto es aprender a cuidar y a ser cuidado, también en la escuela, y no en espacios específicos de educación moral sino en todas las materias y actividades: fomentando la atención al otro, la preocupación por él, una motivación centrada en sus necesidades, una respuesta positiva a quien nos cuida, un clima de confianza que facilita la expresión de sentimientos y deseos. De modo 
que se acaben imbricando saber ser cuidado, cuidarse y cuidar, en la conciencia de lo mucho que aporta a la vida moral también el que es cuidado - aquí, el educando-.

He adelantado que la propuesta de Noddings se confronta sobre todo con el modelo de educación en virtudes. Añado ahora que, como puede intuirse, tiene también algunos toques levinasianos. De todos modos, no debe ser confundida con la propuesta de Levinas, pues los supuestos básicos de ambas son claramente distintitos. Señalo algunas de estas diferencias fundamentales: 1) en Noddings domina el horizonte de simetría en el cuidar y el ser cuidados mientras que en Levinas, la asimetría entre el otro que nos impacta y el yo receptor del impacto es insuperable; 2) en la primera, los afectos ligados a la inclinación al cuidado natural, como la simpatía, son claves y motivan decisivamente la iniciativa del cuidado, mientras que en el segundo, más que los afectos propios, lo que cuenta es «ser afectados» por el otro, algo que se expresa primariamente como «traumatismo»; 3) en la primera, el cuidado busca la desculpabilización, mientras que en el segundo, de la exposición del otro al uno mismo emana una «acusación» (en su sentido amplio de "acusativo») en la que cabe la culpabilidad.

\subsection{Enfoque del cuidado y víctimas}

Sin entrar a analizar en detalle la propuesta, paso a confrontarla con lo que puede suponer para ella que la presencia de las víctimas se convierta en una referencia decisiva. El modelo contiene aspectos facilitadores de esta presencia: la relevancia de los afectos, la narratividad, la interdependencia relacional, la atención a las necesidades del otro y a la vez la disposición a recibir de él, etc. Pero hay otros que parecen bloqueadores, ante los cuales la presencia focal, no circunstancial, de la víctima interpelante, no solo puede producir el desbloqueo y hacer justicia a la víctima, sino que puede reconfigurar positivamente el modelo.

En el enfoque descrito del cuidado se prioriza la referencia al cuidado natural, espontáneamente armonioso. $Y$ se quiere preservar todo lo posible. Por ejemplo, relativizando el mal. Ante quien lo comete, se pide que se enfatice lo bueno que es y hace y que se le atribuya la mejor intención en el mal que causó (nada se dice de la víctima que lo sufrió), pues, se indica, la acusación aleja a quien cuida de quien recibe cuidado, debilita la relación y el diálogo, bloquea la asunción de responsabilidad e implica relación de autoridad- 
subordinación, incluso si está abierta al perdón —que no es apreciado en el modelo- . Pienso que hay modos de condenar ciertas faltas en la cotidianidad de un aula que inducen realmente a eso, por lo que hay que evitarlos. Pero ello no debe conducir a la sola propuesta de diluir el mal y la culpabilidad, debe empujar a afrontarlos ajustadamente. La presencia de la víctima lo pone crudamente de manifiesto: cabe el mal, cabe la culpabilidad que, en efecto, rompe la relación. ${ }^{10} \mathrm{La}$ víctima es la prueba; si eso no se asume, ella no es ni se siente acogida. ¿Supone esto quiebra del modelo relacional? No. Es un imponente reto para reconfigurarlo de modo que contemple como parte de él la recomposición de las relaciones en su sentido fuerte, precisamente desde el reconocimiento de la responsabilidad que puede llegar a culpabilidad y, entonces, de los procesos de arrepentimiento-perdón (se llamen o no de ese modo). Estos, hechos con autenticidad y enmarcables en la «justicia restaurativa», frente a la duramente retributiva que perpetúa la ruptura, son una extraordinaria expresión de relación de cuidado. Donde mejor se muestra pedagógicamente esta posibilidad es en el testimonio de la víctima que se abre a esas reconstrucciones moralmente asimétricas de relación con su victimario. ${ }^{11}$

Esta misma referencia al cuidado natural, ahora como fundamentador de la ética, vuelve a ser convulsionada, desde otra perspectiva, por la presencia de la víctima. ¿Se hizo el mal porque se rompió la relación? Ella piensa más bien que esto fue la consecuencia. Se hizo el mal porque alguien quebrantó su dignidad de persona. Es necesario que el modelo relacional asuma esto, para aportar a continuación lo más propio suyo: afirmar tal dignidad amparándola en la relación de cuidado, cargándola así de sustento afectivo y apoyo concreto. Con este supuesto, la expansión de dinámicas de cuidado más allá de la relación del cara a cara - uno de los puntos débiles del modelo- a desconocidos de los que, de todos modos, consta que han sufrido violación de su dignidad como los cercanos conocidos, pasa a tener más consistencia.

10 Debe matizarse esto aclarando que en este modelo cabe trabajar con historias de víctimas. De hecho, Noddings (2009, 96-101) pone el impactante ejemplo de Wiesenthal, convocado por el nazi moribundo que le victimó, que quiere su perdón. De todos modos, lo que propongo aquí es que estos relatos no aparezcan como algo posible, dejando además el mal fuera del aula, sino de forma tal que la vitimación se convierta en foco decisivo de la educación moral.

11 Aparece aquí un tema muy amplio, además difícil, por estar lleno de malentendidos. Un ejemplo palmario de lo que supone, en este caso para las víctimas del terrorismo, se encuentra en Rodríguez Pascual (2013). Se concreta más expresamente la dimensión moral del mismo, con carga pedagógica, en la aportación de Etxeberria «Los encuentros restaurativos como diálogo moral». 
Una tercera consideración se remite al hecho de que, si inicialmente el cuidador (educador) se hace cargo del que es cuidado (educando), también este aporta al primero (a su educación). Cuando aparece una víctima externa a las relaciones del aula (incluyendo las de los alumnos entre ellos), este esquema se complejiza: quien no estaba en la relación irrumpe en ella, implicando relación temporal pero que deja huella permanente; quien aparentemente necesita ser cuidado en su condición de víctima, se muestra nuestro cuidador en nuestra maduración moral; aunque también él o ella saldrán cuidados, fortalecidos por nuestra receptividad, por el diálogo compartido a partir de su interpelación testimonial. Magnífica depuración e intensificación de la interdependencia que oportunamente se resalta en este modelo.

\section{El modelo de educación del carácter moral y las víctimas}

\subsection{El enfoque del carácter moral}

Es el más clásico, con Aristóteles como una referencia que sigue siendo decisiva, aunque no deban ignorarse las vicisitudes de una larga tradición que le han permitido, más allá de sus miserias, estar renovadamente vivo. Como sabemos, se centra en la adquisición y vivencia de las virtudes o «modos de ser» del carácter moral. Para presentarlo, es oportuno remitirse a Aristóteles (1988) revisado y actualizado, porque es la mejor forma, pienso, de afrontar críticas al modelo que considero extrañas en cuanto ajenas a lo que es, como el reproche de que da por establecidos, claros y unívocos los contenidos de los hábitos virtuosos que se pretenden inculcar, ignorando la singularidad de las personas y de sus situaciones (Puig Rovira 1995), y remitiendo además impositivamente a las propuestas de comunidades culturales fuertes (Nodding 2009). Lo que supondría que es una mera versión del modelo de socialización aquí rechazado.

Recordemos que virtud es, para Aristóteles, un modo de ser, que se precisa como término medio entre dos extremos, elegido con la adecuada deliberación. Lo propio de ese modo de ser es que expresa la realización positiva de lo que podemos ser, nuestra areté o excelencia, unificando en torno a él nuestro psiquismo; que afecta en especial a las emociones, hasta el punto de que podemos considerar las virtudes como modulaciones estables de los sentimientos - que aportan la motivación inherente a ellas - orientados hacia el bien; que, como tales, permanecen en el tiempo, a pesar de las adversidades y en la diversidad de contextos, recontextualizándose; 
que, aunque primariamente no son propiedad de las acciones sino del sujeto, implican disposiciones permanentes -actitudes, se tiende a decir hoy - hacia el bien que nos capacitan para las acciones, que nos conducen con constancia a ellas, que se autentifican en ellas: es aquí donde hay que situar la virtud como hábito —es solo una de sus dimensiones-, pero si no supone rutina ni conformismo.

En cuanto a la virtud como mesotés, solo quiero recordar que es término medio no en cuanto a la excelencia sino en cuanto al contenido de las emociones y las acciones, y que se precisa contextualizadamente, con la guía de la prudencia. Y el que requiera elección deliberada significa que no hay virtud, aunque haya hábito de conducta, si este lo es por mera inclinación natural. Se necesita elegirla expresamente como bien, siguiendo el deseo (atracción y motivación) deliberado (conformado por la razón). Ciertamente en Aristóteles su concepción del ser humano en la polis le empuja a un comunitarismo fuerte, pero siembra las semillas para poder flexibilizarlo.

Que las virtudes tengan concreciones contextuales, personales y culturales, lo muestra su historia (Bollnow 1969): cada vez que hay una transformación cultural hay una transformación en las virtudes propuestas (piénsese en los grandes cambios entre virtudes aristocráticoguerreras de la antigüedad, virtudes burguesas del capitalismo, virtudes románticas, etc.; o virtudes en culturas indígenas, etc.). Esto es, toda virtud tiene una huella histórico-cultural y el reto está en mostrar que no se trata de relativismo denso, localizando en ellas núcleos de disposiciones humanas fundamentales que se realizan parcial y contextualizadamente, con sus logros y sus límites -inherencia en ellas del momento crítico-, en las distintas situaciones de la historia. Lo que se transforma en desafío para reconfigurarlas creativamente en nuestras circunstancias de hoy, desde nuestro sentido de la libertad y la justicia asentados en la dignidad y expresados en los derechos humanos.

Por último, se sabe que ya los griegos debatieron si las virtudes pueden enseñarse. Yo diría que la realidad muestra lo que nos importa, que pueden aprenderse. Y que para ello se han ido detectando vías relevantes como la de la imitación personalizada de personas ejemplares, la vía narrativa de los relatos o la de la educación de los sentimientos.

\subsection{Enfoque del carácter moral y víctimas}

Al enfoque del carácter a lo primero que le retan las víctimas es a que, desde la capacidad que tiene, se actualice en la definición de 
virtudes (reconfigurando las clásicas pertinentes e innovando) y, en sus procesos educativos, haciéndose firmemente cargo de la emergencia hodierna de las víctimas como referencia central para la justicia y la ética en general. Apunto aquí algunas pistas de lo que ello puede suponerle.

En este enfoque ético lo propio de los principios con vocación de universalidad es que se vivan interiorizados como disposiciones hacia su cumplimiento, alcanzando así su verdadera fortaleza. De todos modos, como se ve en Aristóteles cuando se ocupa de la justicia, hay dificultades en expresarlos como meras virtudes, orientadas como tales a la vida buena, al implicar siempre una obligatoriedad que se nos impone. Hay así una tensión, llamada a ser creadora, entre la vertiente externa impositiva del principio y la interna vivenciada. Cuando el enfoque de la virtud asume la perspectiva de las víctimas, debe traducirse en la plasmación de esa tensionalidad positiva en los referentes del respeto a la dignidad y la justicia, que nos permiten discernir dónde hay víctimas y de qué tipo de víctimas se trata, supuesto básico para la acogida de estas, sin que eso pida renunciar a su expresión como virtud. Es algo fácil y fecundamente expresable, en el caso del respeto, como virtud del respeto. En cuanto a la justicia habrá que incluir actualizadamente su expresión distributiva y su expresión penal, la primera, acorde con los derechos sociales, la segunda, discerniendo entre la versión retributiva y la restaurativa, sobre lo que haré un apunte en el apartado siguiente. Ninguna expresión de virtudes deberá contradecir estos referentes.

En segundo lugar, la respuesta aretológica a la interpelación de las víctimas nos empuja a destacar el cultivo personal y social de las virtudes públicas y también de la dimensión pública de las virtudes que parecen mostrarse privadas (Etxeberria 2012), sobre la base de que allá donde hay víctima moral, aunque se dé en la mayor intimidad, hay dimensión pública.

En tercer lugar, las víctimas nos retan a distinguir entre virtudes de la receptividad y de la actividad, ${ }^{12}$ para dinamizar ante su interpelación prioritariamente las primeras (atención, escucha, humildad, reconocimiento, agradecimiento, admiración...), y alentar las segundas como respuesta (dialogalidad, solidaridad, valentía, justicia, cuidado, etc.), sabiendo que hay virtudes «mixtas» clave como la compasión

12 La distinción la hace Macintyre (2001). Con ella pretende diferenciar virtudes de la independencia y del reconocimiento de nuestra dependencia. Con la perspectiva de las víctimas esto se transforma en virtudes de nuestra «dependencia» y «vulnerabilidad», en sentido levinasiano, ante las víctimas y virtudes de nuestra respuesta a ellas. 
y el respeto. Con ello se acoge el ineludible momento primario de la alteridad de la víctima, sin llegar a su hiperbolización levinasiana.

Esta distinción nos conduce a otro aspecto que debe tenerse en cuenta. Desde el modelo relacional se acusa al enfoque de la virtud de centrarse en el agente. Pero eso no es así cuando se cultivan expresamente virtudes relacionales como las citadas en las que se sintetiza horizonte de excelencia de uno mismo y colaboración en la excelencia del otro, de la víctima.

Otra cuestión clave que las víctimas nos fuerzan a resaltar con su presencia activa es la concepción de la virtud como modulación de los sentimientos. La experiencia nos muestra el papel clave que, cuando no son virtudes, tienen, primero en victimar, luego en revictimar. El mismo papel clave están llamados a tener, como sentimientos virtuosos, en frenar esas victimaciones expresando la potente contrapartida de la empatía que acoge. Ante la víctima, en efecto, se ha manifestado odio y menosprecio, acrecentados por la admiración al victimador. Frente a ello, el enfoque de la virtud pide que imbriquemos tres sentimientos virtuosos: el de la indignación ante lo hecho por el victimario, el de la compasión ante lo sufrido por la víctima, el del respeto para expresar ambos en su modo adecuado.

Por último, la presencia educadora activa de las víctimas implica per se una intensa práctica de las vías propias de este modelo que se muestra pedagógicamente muy fecunda: la ejemplaridad testimonial, la narratividad, la educación de los sentimientos.

\section{El modelo del desarrollo del juicio moral y las víctimas}

\subsection{El enfoque del juicio moral}

En la medida en que se considere la versión de Kohlberg (1981), con sus enraizamientos kantianos y rawlsianos y precedida por la de Piaget, como canónica —es lo que aquí voy a presuponer-, nos encontramos con un modelo muy preciso y con gran coherencia interna.

Recordemos sus supuestos básicos: 1) La educación debe centrarse en el desarrollo de la capacidad de juicio moral racionalmente sustentado, frente a la atención a los aspectos emocionales, motivacionales y conductuales; 2) ese desarrollo es plasmado en estadios que expresan una progresiva maduración cognitiva moral, estimulándose al educando a recorrerlos; 3 ) los referentes morales en juego se nuclean en torno a los principios formales de justicia equitativa, percibidos con validez universal 
y llamados a guiar las decisiones; 4) tienen como sustrato la concepción liberal del individuo humano sujeto de una dignidad de la que emanan derechos; 5) pedagógicamente, se privilegia el debate en torno a dilemas morales que reflejan situaciones conflictivas de la vida, de modo tal que se fomente la argumentación racional sobre lo justo; 6) implicando diálogos que, realizando una comunidad democrática en el aula y el centro escolar, educan para la democracia en la sociedad.

Como ya se sabe bien, se contemplan seis estadios en tres niveles de fundamentación de los juicios: el preconvencional, en el que cuentan las consecuencias físicas y hedonistas; el convencional, en el que se busca la conformidad con la autoridad y los grupos de pertenencia; el postconvencional, en el que priman la actitud básica de la autonomía, frente a las heteronomías precedentes, y la búsqueda de validez universal de los juicios morales.

Tampoco aquí voy a entrar en el debate en torno a esta propuesta, ya muy conocido (Ortega y Mínguez 2005), sino que me centraré en señalar en esbozo cómo queda conmocionada si más allá de los dilemas minuciosamente preparados para estimular los debates, se confronta a los estudiantes con el testimonio de las víctimas. Permítaseme antes, con todo, algún detalle evaluativo. Frente a ciertas críticas, no debe ignorarse que Kohlberg es consciente de que el desarrollo del pensamiento moral, siendo para él indispensable, es incompleto si no acaba en la acción. Esto puede abrir a imbricar su propuesta con elementos de otros modelos -o con lo que supone el revulsivo de las víctimas-, en la conciencia, con todo, de que ello supondrá reconfigurar la concepción de algunos de sus supuestos -al menos su rigidez-. Es cierto que ante tal tarea nos encontramos con un menosprecio kohlbergiano de los contenidos de la moralidad, percibidos como "saco de virtudes», que deberá ser superado; aunque haya que tener presente que su consideración negativa de ellas parece estar sustentada en propuestas de pedagogos de la educación del carácter que las conciben, inadecuadamente, como rasgos de la personalidad sancionados por la sociedad.

\subsection{Enfoque del juicio moral y víctimas}

Que en los educandos las confrontaciones decisivas para su desarrollo moral cognitivo se den con víctimas que les interpelan, puede aportar a este enfoque algo muy valioso. Esas víctimas, en efecto, dan «evidencia de experiencia» a los principios de justicia en el crudo quebrantamiento de ellos, situando además el mal en su lugar real, 
no en el incumplimiento per se de un imperativo sino en el daño a la persona en su dignidad y en ella a la sociedad: hay imperativo porque puede haber daño y para que no lo haya. A su vez, la argumentación racional tiene su momento, aunque ahora actúe reactivamente, para definir ajustadamente, como señalé antes, qué entender por víctimas y qué deberes se nos imponen ante ellas.

Las víctimas, además, fuerzan a atender principios de justicia que tienen que ver no solo con la distribución de bienes (a lo Rawls) sino con la reacción ante el mal moral hecho, que en general se descuida en la educación. En este sentido, interpelan a este modelo - y a todospara que revise la concepción kantiana duramente retributiva de la justicia frente al delito —la de la ley del talión «desentimentalizada»-. Las víctimas, en su pluralidad reactiva ante sus victimarios, lo abrirán a este debate importante, haciendo aparecer una pregunta clave: ¿debe la justicia tener un horizonte no de retribución sino de sanación de todos los implicados por el mal, en procesos asimétricos en función de la inocencia y la culpabilidad, como se propone desde su enfoque restaurativo? ${ }^{13}$

Otra aportación crítico-transformadora: las víctimas que testimonian «nos conmueven», ponen en primer plano las emociones. Si lo hacen con los supuestos educativos descritos, muestran que son plenamente válidas y marcadamente valiosas. En la propia experiencia de vivirlas, ya no se perciben enfrentadas a los principios, sino dándoles fuerza y motivación. E incluso pueden ser fuente de conocimiento, de que hagan emerger realidades no localizables espontáneamente por la razón. ${ }^{14}$ Son también un riesgo serio, pero que no convoca a relegarlas sino a educar expresamente en ellas, para que se ajusten a los principios y a la vez los ajusten y, de ese modo, su fuerza motivacional quede bien encauzada.

Que el proceso educativo arranque con la receptividad de la interpelación de la víctima ayudará igualmente a que se module la concepción de la autonomía moral, tan central en este modelo. No puede ni debe ser la supuestamente ideal autonomía de la persona autosuficiente, pues el ser humano está siempre en posición de deudor de otros, aquí, de las víctimas. Se ha dicho, creo que con razón, que la autonomía consciente de lo que recibe y que se expresa como responsabilidad —es nuestro caso- puede ser definida, paradójicamente, como síntesis de contrarios, como «heteroautonomía» (Rogozinski 1999).

13 Puede verse un análisis evaluativo de los diversos enfoques de la justicia ante el delito, con apuesta final por el modelo restaurativo, en Etxeberria (2014c).

14 Véase Nussbaum (2015). 
Por último, la complejidad de la respuesta a la interpelación de las víctimas, que pide hacerse cargo de sus contextos, muestra a este modelo que, junto a la racionalidad principal, tendente a aplicaciones rígidas que pueden incluso llegar a causar víctimas, tenemos la racionalidad prudencial de tipo aristotélico, enormemente atenta a las circunstancias y consecuencias que hay que saber activar, sin que se identifique con el utilitarismo potencialmente justificador de chivos expiatorios.

\section{Conclusión: el revulsivo transversal de las víctimas}

El corazón de la aportación de las víctimas, cuando hacen acto de presencia como educadoras en los procesos de formación moral en las aulas es, como indiqué, el testimonio de lo que fue y supuso su victimación, esto es, la violación de sus derechos humanos, con su reacción ante ella, y el diálogo al que ello abre. En esa iniciativa, llamada a dar sentido a todo el recorrido formativo, hay aspectos muy relevantes.

Se dan, como se ve, dos momentos. En primer lugar, aquel en el que se testimonia la presencia del mal. No es evidentemente, como tal, momento ejemplar. Tampoco es en sí meritorio, pues dicho mal a la víctima, haciéndole víctima, le impactó «a su pesar». Pero es educativo de un modo original y efectivo: no se educa a partir de una discutible idea de bien, sino a partir de una experiencia de mal (Bárcena 2003), nada evanescente. En segundo lugar, está el momento que da cuenta de la reacción de la víctima, muy pegado a la realidad y a la vez transmitiendo fuerza para confrontarse con el mal. Si el primer momento iguala a las víctimas como víctimas, en el segundo las personalidades, contextos y procesos de cada una pueden establecer diferencias. Pero en cuanto movidas por el auténtico aliento educativo, todas ofrecen un amplio horizonte con el que confrontarse: es el momento de aprender del bien que nos aportan y de abrirse al diálogo.

Esta experiencia educativa que se vive en el presente remite, como se ve, a un pasado, el del acontecimiento victimador. La víctima nos reclama que le hagamos un lugar fundamental en la educación para el bien, que la memoria que ella tiene de él pase solidariamente a formar parte de nuestra memoria, que lo retrabajemos con ella sin falsearlo para que module un recuerdo que, además de clamar justicia, aliente transformaciones de cara al futuro. Porque está, por último, este futuro, pues la narración de la víctima no es cerrada, abre a él. Y es en él, desde los enraizamientos memoriales, en donde se nos pide nuestra participación activa, en donde se plasma nuestra responsabilidad. 
Pedagógicamente, como he ido apuntando, todo esto supone movilizar dinámicas de narratividad, receptividad, sentimentalidad, reflexividad, responsabilidad, dialogalidad. Es decir, supone alentar y renovar las mejores vías de educación moral.

Una presencia así de las víctimas, como se ha visto, es posible y necesaria en cualquiera de los modelos fundados de educación moral. Como tal, es transversal a todos ellos. Por todos puede ser amparada. A todos los cuestiona, los afina, los desarrolla. A todos les pide que establezcan puentes con aportaciones de otros modelos en vistas a lo fundamental, que no debe ser situado en que la pureza del modelo se realice sino en que la buena educación se logre. En este objetivo las víctimas habrán tenido un papel relevante, que toca a los educadores promover, reconocer y agradecer.

Acabo subrayando un aspecto que solo puntualmente he señalado: a través de este proceso educativo focalizado en las víctimas y centrado en su iniciativa, se realiza en los estudiantes, con gran carga vivencial, una iniciación en lo nuclear de los derechos humanos. En efecto, y en primer lugar, estos derechos no han surgido de propuestas reflexivas abstractas hechas por grandes pensadores; han emergido, en forma de indignación, en personas de todas las culturas que, al sufrir violencias de otros o al verlas en sus semejantes, han percibido en progresivas clarificaciones que eran injustas habida cuenta de su condición de humanos, y han gritado, de un modo u otro: "ino hay derecho!». Dicho de otra forma, la concepción de la justicia de los derechos se ha ido perfilando desde las experiencias de injusticia (Renault 2004; Mate 2011). Pues bien, con la presencia testimonial de las víctimas, alumnos y profesores se introducen por empatía en esta vivencia. Es sobre ella como pueden captar luego con sentido y convicción la construcción reflexiva de los derechos humanos. En segundo lugar, estos derechos alcanzan toda su consistencia cuando, viendo a los otros como sujetos de ellos, asumimos efectivamente los deberes que evocan en nosotros hacia esos otros. Es lo que sucede también con las víctimas educadoras: su intervención en los procesos educativos hace efectivo su derecho a ser reconocidas como tales, derecho básico en cuanto que es condición de posibilidad de todos los demás. En los centros educativos son reconocidas del modo más pleno, en el protagonismo que se les posibilita. Y el reconocimiento lo expresa no solo la institución, sino los propios estudiantes al escucharles: a la vez que aprenden, reconocen. Para las víctimas - lo sé por la experiencia realizada- es algo enormemente valioso. 


\section{Referencias bibliográficas}

Arana, Ricardo y García, Mónica. 2012. Las víctimas en la educación. Un testimonio de valor. San Sebastián: Gobierno Vasco.

Aristóteles.1988. Ética nicomáquea. Ética eudemia. Madrid: Gredos.

Bárcena, Fernando. 2003. "La mirada excéntrica. Una educación desde la mirada de la víctima», en La ética ante las víctimas, editado por José M. ${ }^{a}$ Mardones y Reyes Mate, 195-218. Barcelona: Anthropos.

Bilbao, Galo y Xabier Etxeberria. 2005. La presencia de las víctimas del terrorismo en la educación para la paz en el País Vasco. Bilbao: Bakeaz.

Bilbao, Galo, Cristina de la Cruz y Pedro Sasia. 2010. Víctimas: todas iguales, todas diferentes. Bilbao: Bakeaz.

Bollnow, Otto F. 1969. Esencia y cambios de las virtudes. Madrid: Revista de Occidente.

Etxeberria, Xabier. 2012. Virtudes para convivir. Madrid: PPC.

Etxeberria, Xabier. 2013. La educación para la paz reconfigurada: la perspectiva de las víctimas. Madrid: Catarata.

Etxeberria, Xabier. 2014a. «El valor de la presencia de las víctimas del terrorismo en la educación vasca». Cuadernos de Pedagogía 445: 86-90.

Etxeberria, Xabier. 2014b. «El debate ético sobre la dignidad de la persona». Lumen, LXIII, n. 2-3: 229-249.

Etxeberria, Xabier. 2014c. «En torno a la legitimidad y el sentido del castigo por el delito». Revista portuguesa de filosofía 70, n. 4: 765-786.

Galanti, Sebastiano. 2013. "Riconoscimento e sensibilità: II conflitto con I'altro tra Ricoeur e Levinas». Archivio di Filosofia LXXXI, n. 1-2: 27-35.

Harillo, Susana, Jesús Prieto y Josu Ugarte. 2006. Historias que nos marcan: las víctimas del terrorismo en la educación para la paz. Guía didáctica para educación secundaria. Bilbao, Bakeaz. Consultado el 6 de abril de 2015. Disponible en: www.hezkuntza.ejgv.euskadi.eus

Kant, Immanuel. 1973. Fundamentación de la metafísica de las costumbres. Madrid: Espasa Calpe.

Kohlberg, Lawrence. 1981. Essays on Moral Development. The Philosophy of Moral Development. Stages and the Idea of Justice. San Francisco: Harper and Row.

Levinas, Emmanuel. 1987. De otro modo que ser o más allá de la esencia. Salamanca: Sígueme.

Levinas, Emmanuel. 1991a. «Transcendence et hauteur», en Emmanuel Levinas, dirigido por C. Challier y M. Abensour, Paris: de l'Herne.

Levinas, Emmanuel. 1991b. Ética e infinito. Madrid: Visor.

Levinas, Emmanuel. 1991c. Entre nous. Paris: Biblio essais.

Levinas, Emmanuel. 1994. Dios, la muerte y el tiempo. Madrid: Cátedra.

Macintyre, Alasdair. 2001. Animales racionales y dependientes: por qué los seres humanos necesitamos las virtudes. Barcelona: Crítica.

Mate, Reyes. 2011. Tratado de la injusticia. Barcelona: Anthropos.

Mínguez, Ramón. 2010. "La escuela hoy en la encrucijada. Hacia otra educación desde la ética de E. Levinas». Teoría de la Educación 22: 43-61. 
Mínguez, Ramón, Eduardo Romero y Marina Pedreño. 2016. «La pedagogía del otro: Bases antropológicas e implicaciones educativas». Teoría de la Educación 28, n. 2: 163-183.

Noddings, Nel. 2009. La educación moral. Propuesta alternativa para la educación del carácter. Buenos Aires: Amorrortu.

Nussbaum, Martha. 2015. Paisajes del pensamiento. La inteligencia de las emociones. Barcelona: Paidós.

Ortega, Pedro. 2004. "La educación moral como pedagogía de la alteridad». Revista española de pedagogía LXII, n. 227: 5-30.

Ortega, Pedro y Ramón Mínguez. 2005. "La educación moral, ayer y hoy». Revista Galega do Ensino 13, n. 46: 863-885.

Ortega, Pedro y Eduardo Romero. 2013. "La experiencia de las víctimas en el discurso pedagógico». Teoría de la educación 25, n. 1: 63-77.

Ortega, Pedro. 2016. «La ética de la compasión en la pedagogía de la alteridad». Revista española de pedagogía, LXXIV, n. 264: 243-264.

Puig-Rovira, Josep M. ${ }^{a}$ 1995. "Construcción dialógica de la personalidad moral». Revista Iberoamericana de Educación, 8: 103-120.

Renault, Emmanuel. 2004. L'expérience de l'injustice. Paris: La Découverte.

Ricoeur, Paul. 1990. Soi-même comme un autre. Paris: Seuil.

Rodríguez Pascual, Esther. (coord.) 2013. Los ojos del otro. Encuentros restaurativos

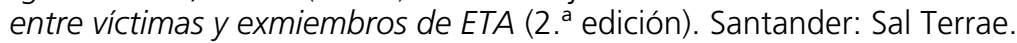

Rogozinski, Jacob. 1999. Le don et la loi. Paris: PUF.

Santos, Marcos. 2012. "Pedagogía y memoria. Educar a partir del recuerdo de los vencidos», Pensamiento 68, n. 255: 79-106. 


\section{Copyright}

Deusto Journal of Human Rights / Revista Deusto de Derechos Humanos is an Open Access journal; which means that it is free for full and immediate access, reading, search, download, distribution, and reuse in any medium only for non-commercial purposes and in accordance with any applicable copyright legislation, without prior permission from the copyright holder (University of Deusto) or the author; provided the original work and publication source are properly cited (Issue number, year, pages and DOI if applicable) and any changes to the original are clearly indicated. Any other use of its content in any medium or format, now known or developed in the future, requires prior written permission of the copyright holder.

\section{Derechos de autoría}

Deusto Journal of Human Rights / Revista Deusto de Derechos Humanos es una revista de Acceso Abierto; lo que significa que es de libre acceso en su integridad inmediatamente después de la publicación de cada número. Se permite su lectura, la búsqueda, descarga, distribución y reutilización en cualquier tipo de soporte sólo para fines no comerciales y según lo previsto por la ley; sin la previa autorización de la Editorial (Universidad de Deusto) o la persona autora, siempre que la obra original sea debidamente citada (número, año, páginas y DOI si procede) y cualquier cambio en el original esté claramente indicado. Cualquier otro uso de su contenido en cualquier medio o formato, ahora conocido o desarrollado en el futuro, requiere el permiso previo por escrito de la persona titular de los derechos de autoría. 